\title{
Brown fat tissue - a potential target to combat obesity
}

\author{
Ginter $E^{1}$, Simko $V^{2}$ \\ Institute of Preventive and Clinical Medicine, Bratislava, Slovakia. ginter.emil@mail.t-com.s
}

\begin{abstract}
From the global population perspective, the epidemic of "globesity" (more than one billion adults being overweight) represents one of the largest public health problems (1). Traditional reasoning related to the dysbalance between caloric intake and energy expenditure does not provide a satisfying explanation for a complex failure to combat obesity. The brown adipose tisue (BAT) has a unique chemical structure and a specific metabolic role. A potential preventive co-factor is thermogenesis. BAT has the ability to dissipate energy by producing heat, rather than storing energy as triglycerides. The cells of the white adipose tissue (WAT) contain one large globule of triglycerides which displaces the cell nucleus and other cell organelles excentrically, to the cell periphery. BAT contains numerous smaller droplets of triglycerides, much higher number of mitochondria and a specific uncoupling protein 1 or thermogenin. This specialized protein uncouples ATP production from mitochondrial respiration and converts energy into heat. Using sophisticated diagnostic techniques (e.g. imaging combination of positron-emisson tomography and computed tomography), scientists confirmed the importance of BAT not only in the newborn but also in adults who were found to possess considerable body stores of BAT. The highest proportion of BAT has been detected in lean individuals. As the body mass increases, BAT proportionately drops. Data both from animal and human studies suggest that BAT and mitochondrial uncoupling can be targeted for interventions to prevent and treat obesity. Melatonin and arginine have been proposed as possible interventional tools. The scientific world eagerly awaits further advanced studies to document possible metabolic and pharmacologic interventions, using BAT as a primary target to prevent and manage obesity (Fig. 5, Ref. 41). Full Text in free PDF www.bmj.sk.

Key words: brown adipose tissue, thermogenesis, cellular bioenegetics, mitochondria, uncoupling protein 1 , adipose tissue engineering, obesity prevention.
\end{abstract}

A simplified view is that overweight and obesity result from energy imbalance. However, the worldwide increase in obesity indicates there is a losing battle against this widely prevalent metabolic disorder. Only less than one third of the US adults are at a healthy weight (2). About every third person in the United States who is older than 20 is obese (body mass index, BMI $\geq 30$ ) and almost 6 per cent are afflicted with extreme obesity $(B M I \geq 40)$ (3). Example: a man of $175 \mathrm{~cm}$ height who is extremely obese weighs over 123 kilograms.

Obesity is prevalent in many parts of the world. The oil-rich countries (Kuwait, United Arab Emirates and Saudi Arabia) as well as Europe (Czech Republic, United Kingdom, Germany) are no exception (4). Obesity, especially its extremes is associated with an increased risk of diabetes, hypertension, cardiovascular disorders, degenerative joint disease, decreased resistance to infection and some types of malignancy $(4,5)$. All this is prompting an intensive search to moderate and to decrease the risk of obesity.

A common view on adipose tissue is associated with a yellowish- white mass that cushions mostly the subcutaneous space

${ }^{1}$ Institute of Preventive and Clinical Medicine, emeritus, Bratislava, Slovakia, and ${ }^{2}$ State University New York, Downstate Medical Center at Brooklyn, USA

Address for correspondence: E. Ginter, RND, DSc, Racianska 17, SK83102 Bratislava, Slovakia. and the abdominal cavity. Fat represents up to 20 per cent of body mass in men and 25 per cent in females. The adipocytes of the WAT are cells with a single large triglyceride globule, in the BAT adipocytes the triglycerides are dispersed in smaller droplets. Excess accumulation of WAT results in obesity $(\mathrm{BMI} \geq 30)$.

Obesity is a complex condition with many causal contributors, including genetic ones and many environmental factors. Within the brain, the hypothalamus is an important regulator of energy homeostasis (6). Better understanding of the metabolic role of BAT, especially in the last two years, triggered novel attempts to manage obesity more effectively.

BAT in contrast to WAT has much different structure and metabolism (7). Human BAT is unique in that it has evolved to safely dissipate large amounts of chemical energy, a quality that might be harnessed to help humans to deal with a consistently hypercaloric environment and still remain in good health. BAT is especially abundant in newborns and in hibernating mammals because its primary function is to generate body heat in newborns.

\section{BAT, its localization, chemical structure and lipid oxidation}

In small mammals BAT is essential for the maintenance of body temperature and possibly to protect against adverse consequences of excessive energy uptake (8). Activation and/or expansion of BAT counteracts metabolic consequences of diet-induced 
ACTIVE BROWN ADIPOSE TISSUE IN ADULT HUMANS

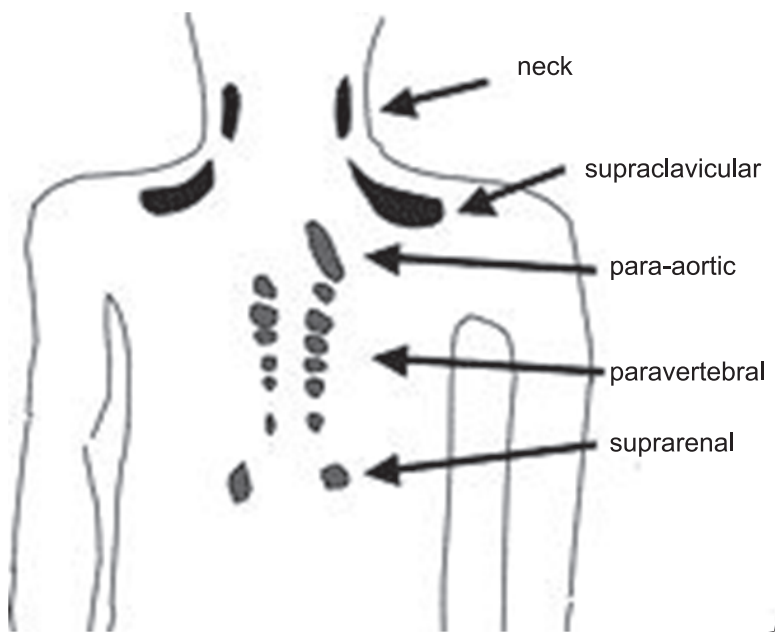

Fig. 1. Distribution of BAT in adult humans. According to Nedergaard et al (12)

body weight gain. BAT has been documented in adult humans to be negatively associated with the BMI. BAT body content decreases with aging (9). It is abundant during the embryonic and early postnatal life, substantially decreasing during later life. Relatively small amount of BAT is located in paracervical and supraclavicular regions (Fig. 1). Essential data on BAT body distribution was obtained by using fluorodeoxyglucose with positron emission tomography combined with computer tomography (PET/CT). The detection of BAT, like of some other metabolites, depends on the diagnostic accuracy. This is still a matter of controversy. Detection of BAT by PET/CT may have a variable reproducibility. Therefore it is advisable to perform more scans than just one: a single scan may underestimate the presence of BAT (10). Also, BAT may exist in adipocytes disseminated throughout the body and this may cumulatively represent substantial BAT activity, not detectable by $\mathrm{PET} / \mathrm{CT}$ and other crude biochemical assays (11). Gene and protein expression analyses promise to further increase the sensitivity of BAT detection $(12,13)$.

More sensitive diagnostic techniques put to rest previous claims that diet-induced thermogenesis is a myth (14). Individuals with a low content of BAT are prone to be overweight and obese, have insulin resistance and cardiovascular disorders. Restriction of dietary calories promotes BAT activity and it may protect the organ systems against premature aging (9). BAT activity in the human body documented by PET/CT exhibits marked seasonal variations (15).

BAT participation in metabolic regulations raises the possibility for adipose tisue to be considered a component of the hormonal system. Adipose tissue secretes a number of bioactive proteins collectively designated as adipokines. These include leptin, adiponectin, resistin, tumor necrosis factor alpha and interleukin 6. These are bioactive proteins influencing insulin resistance and protection against inflammation.
While metabolic impact of sex homones (estrogen, testosterone) on obesity has been long known, the hormonal role of BAT related to male or female gender has only recently been suggested (16). BAT is more prominent in younger people and in female subjects. With age, BAT declines disproportionately more in males. Male sex, compared to females is notorious for more cardiovascular disease and shorter life expectancy.

Regulation of body temperature depends on the integrated coordination of the autonomic nervous system, predominantly the part of brain called hypothalamus. PET/CT scans of subjects with active BAT documented significant hypermetabolic area in specialized parts of human brain (17).

Neuronal pathways within the hypothalamus that are critical in controlling feeding and energy expenditure in relation to BAT, have been identified and their peptidergic content was characterized (18). Endogenous actions of such peptides indicate a role for orexigenic and anorexigenic hypothalamic peptides that influence the thermogenic balance via BAT activity.

In newborn infants BAT represents about 5 per cent of the body mass. For infants BAT is very essential to avoid critical body cold. Previous skepticism on the thermogenic role of BAT in adults, based on relative paucity of brown adipocytes was subsequently changed $(14,19)$. Over recent past years, several investigators using more sensitive diagnostic tools, PET/CT imaging, immunohistochemistry, gene and protein expression assays, revealed significant sites of metabolically active BAT in adult humans $(12,13,20)$.

Each type of fat, WAT or BAT has a unique developmental gene expression which is largely independent of the nutritional state (21). This suggests a fundamental role for developmental genes in fat distribution, development and future function of adipose tissue depots. Formation of BAT begins with stimulation of preadipocytes by expression of the PRDM16 gene to differentiate these into BAT cells. PRDM16 protein causes increased expression of mitochondrial genes and of the uncoupling factor UCP1 (22).

In contrast to white fat cells, BAT cells contain numerous smaller droplets of triglycerides and a much higher number of mitochondria, which contain iron and make it brown (Fig. 2). BAT contains more capillaries than the white fat, since it has a greater need for oxygen than most tissues.

Abnormal fat accumulation and obesity depend to a large degree on the extent with which the body metabolizes lipids. Oxida-

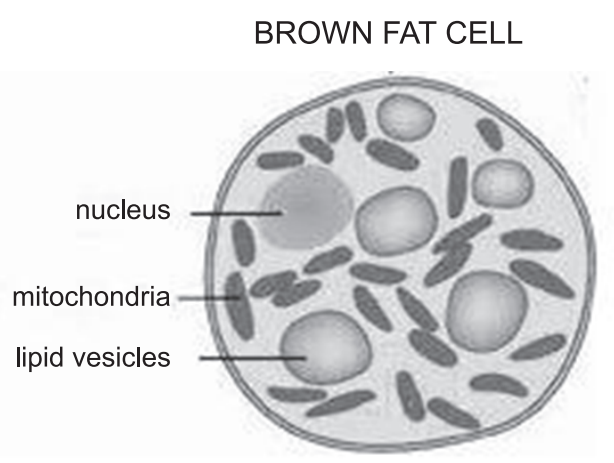

Fig. 2. Structure of BAT cell. 
tion of lipids in the mitochondria of skeletal muscle, in WAT and in several other tissues is a complex process. The fatty acids are broken down in a cyclic process to acetyl-CoA by beta-oxidation. Each round of this cycle reduces the length of the acyl chain by two carbon atoms and produces ATP by oxidative phosphorylation. Dozens of ATP molecules can be generated by the beta-oxidation of a single long acyl chain.

Lipid oxidation in the BAT proceeds differently. After the sympathetic nervous system is activated, lipolysis occurs in the brown adipocytes as the result of activation of the hormone-sensitive lipase. The free fatty acids are then bound to fatty acid binding proteins and transferred into the BAT mitochondria by the activation of carnitine shuttle system.

BAT synthesizes a specific protein UCP-1 (uncoupling protein 1, thermogenin).This belongs to proteins that are capable to shunt away ATP production from the mitochondrial respiration, thereby converting chemical energy into heat. UCP-1 is a transmembrane protein that discharges the proton gradient generated in oxidative phosphorylation. UCP-1 performs this by increasing the permeability of the inner mitochondrial membrane, allowing protons that have been pumped into the intermembrane space to return to the mitochondrial matrix. UCP1-mediated heat generation in BAT uncouples the respiratory chain, allowing for fast substrate oxidation with a low rate of ATP production.

Expression and the activity of UCP-1 is regulated via the sympathetic autonomous system. Because BAT cells possess large numbers of mitochondria in which oxidative phosphorylation is uncoupled from ATP production, they contribute to rapid oxidation of fatty acids (23).

\section{The role of BAT in obesity prevention}

Effective management of obesity is crucial for the reduction of morbidity due to cardiovascular and other disease. The development of obesity not only depends on the balance between food intake and caloric utilization but also on the balance between WAT, which is the primary site of energy storage, and BAT, which is specialized for energy expenditure $(9,24,25,26)$ (Fig. 3). New exciting possibilities to intervene in the pathogenesis of obesity have been reflected in the number of published scientific reports: In September 2010 the PubMed listed over 8300 articles related to BAT.

It has ben known for several years that transgenic mice with genetic ablation of brown fat develop obesity even in the absence of excessive intake of calories (27). Genetic studies in mice have shown that experimental increase in the amount and/or function of brown fat promotes a lean and healthy phenotype. Studies of rodents in which BAT levels were experimentally increased or decreased have revealed a role for BAT in protection against diet-induced obesity. Data in man suggest that individuals with low levels of BAT are prone to obesity, insulin resistance and cardiovascular disease, whereas those with higher levels of BAT maintain lower body weights and exhibit better health as they age.

Experiments reported in recent years confirm that BAT has a potential also in prevention of obesity. Novel detection techniques (28) determined that functional BAT exists in adult humans with

\section{Body weight maintenance depends on BAT activity}

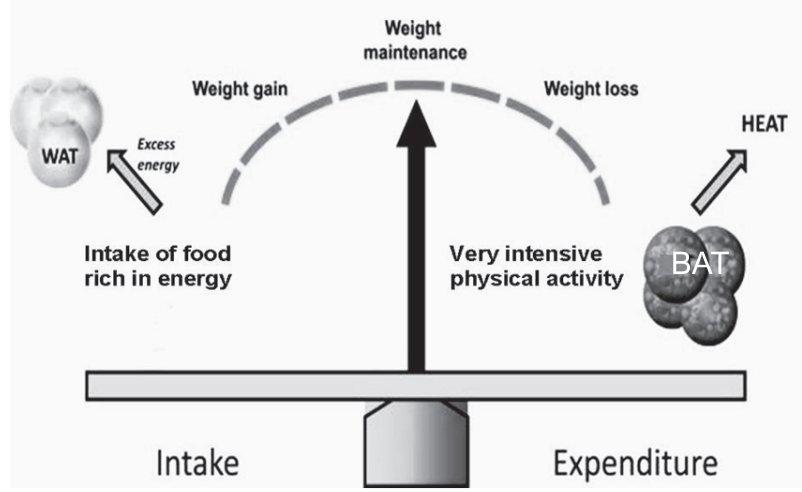

Fig. 3. The role of the white adipose tissue (WAT) and of the brown adipose tissue (BAT) in controlling the body mass. According to Cannon and Nedergaard (26).

\section{Decrease of persons with detectable BAT with increasing age and $\mathrm{BMI}$}
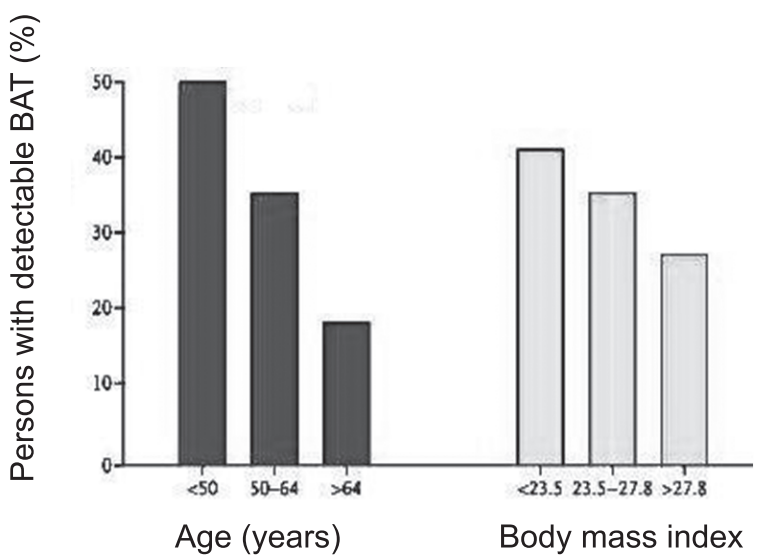

Fig. 4. Decrease of detectable BAT with increase of age and of body mass index in humans. According to Cypess et al (20).

prominent female predominance. BMI is inversely correlated with the amount of BAT, especially in older patients, suggesting a possible role of BAT in protection against obesity (Fig. 4).

Obese individuals have less BAT than lean subjects $(16,29,30)$ which suggests that BAT may be an important factor in maintaining a lean phenotype. BMI and percentage of body fat both display significant negative correlation with BAT. Obese individuals also have a lower level of a decisive protein UCP-1 (uncoupling protein). This protein facilitates direct conversion of the energy from triglycerides into heat (31).

UCP-1 gene locus disorder is a common aspect of reduced UCP-1 gene activity in obese subjects. This leads to a vicious cycle: an individual in energy imbalance increases his BMI. As a consequence, there is a decrease in BAT and in lipid oxidation resulting in pre- obesity. This metabolic condition further reduces the BAT activity, along with lowering the uncoupling protein 1. The result is a decreased fat metabolism, persistence of this process leads to higher degrees of obesity (Fig. 5). 


\section{Circulus vitiosus: high BMI - low BAT - obesity}

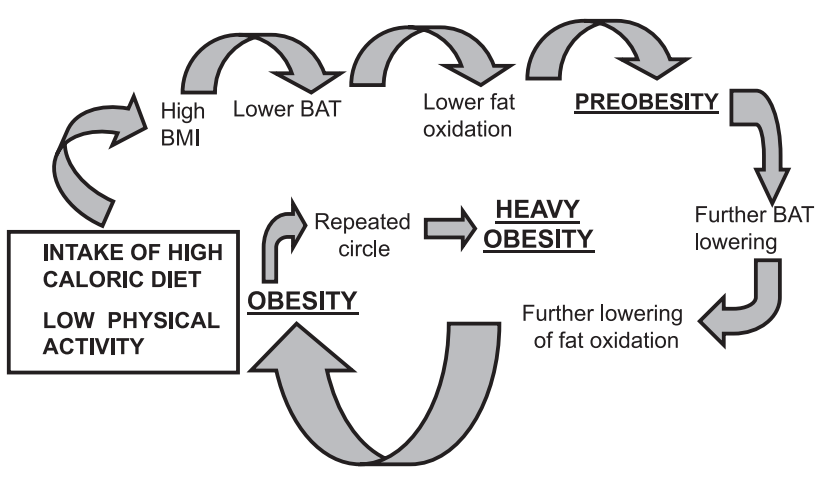

Fig. 5. Hypothetical vicious cycle illustrating the relation between the body mass, the brown adipose tissue and the lipid oxidation.

\section{BAT as a strategy to prevent obesity (novel therapeutic visions)}

Current obesity management focused on reducing the caloric intake and at increasing energy expenditure by exercise indicates that this strategy is not fully effective.

Of the two strategies for generating functional adipose tissue, the first one based on autologous BAT transplantation proved ineffective. Free fat transplants rarely achieve sufficient functional tissue because of deficient neovascularization and cell necrosis (32). The second strategy, fat tissue bioengineering, the use of adult mesenchymal cells and preadipocytes, appears to be more promising. Recent reports suggest that increasing cellular energy expenditure, a bioenergetics approach, may be an attractive alternative (33). Adaptive thermogenesis is the physiological process when energy is utilized in mitochondria of the brown fat and skeletal muscle in the form of heat.

Data both from animal and human studies suggest that BAT and mitochondrial uncoupling can be targeted for interventions to prevent and treat obesity. Although the idea of stimulating BAT activity to combat obesity is a rational approach, it is also conceivable that this may trigger counterregulatory mechanisms such as increased appetite to maintain energy homeostasis and preserve fuel reserves. By uncoupling mitochondrial oxidative phosphorylation, the drug 2,4-dinitrophenol causes a marked increase in fat catabolism that suggested its use to aid weight loss. 2,4-dinitrophenol has historically been used as a herbicide and fungicide. This chemical is quite toxic. Three deaths were reported after ingestion of 2,4-dinitrophenol (34).

Several reports recently indicated other pathways to regulate BAT. One possibiity is the use of melatonin, a lipophilic hormone produced at night by the pineal gland. Melatonin acts via high affinity $\mathrm{G}$ protein-coupled membrane receptors, that also contribute to generation of UCP-1 (35). Both cyclic adenosine monophosphate and cyclic guanosine monophosphate (cGMP) contribute to the intracellular regulation of mitochondrial biogenesis and the differentiation of BAT. New evidence has defined the essential role of the cGMP-dependent protein kinase I in a pathway that modulates insulin receptor signaling, to elicit BAT differentiation and stimulate thermogenesis (36). Recent studies indicate that L-arginine supplementation stimulates mitochondrial biogenesis and BAT development possibly through the enhanced synthesis of cell-signaling molecules (e.g., nitric oxide, carbon monoxide, polyamines, cGMP, and cAMP) as well as the increased expression of genes that promote whole-body oxidation of energy substrates (37).

Mouse experiments confimed that the ApoA-I, the most abundant protein constituent of high density lipoprotein (HDL) has an anti-obesity effect in the mouse. This is associated with increases in energy expenditure and UCP1 expression in the BAT (38).

In a patient with insulin resistance thyroid hormone induced BAT and improvement of diabetes (39). Exciting were the reports that mature human white adipocytes can be converted into brown fat-like adipocytes, and that typical fatty acid storage phenotype of white adipocyte can be altered towards a fat utilization phenotype (40). Included in the reports on the molecular biologic regulation of BAT were data on PRDM16, a zinc-finger protein that controls brown fat determination by stimulating brown fat-selective gene expression, while suppressing the expression of genes selective for white fat cells (41).

\section{Conclusion}

Over the past few years, especially after 2008 our knowledge of development and metabolic role of BAT in humans has increased substantially. Molecular mechanisms underlying brown adipocyte development and their origin from muscle precursors, have been revealed. There is evidence that mature white adipocytes, important in the pathogenesis of obesity, can be converted into brown fat-like adipocytes with distinctly different metabolic function. Thus the typical fatty acid storage phenotype of white adipocytes can be converted toward a fat utilization phenotype. Several possible targets that may be useful for the expansion and/or activation of BAT by physiological and pharmacological means have been identified. Whether we entered an era of successful engineering of brown fat in the battle against obesity remains to be seen $(8,23,40,41)$.

\section{References}

1. Hansen JC, Gilman AP, Odland JO. Is thermogenesis a significant causal factor in preventing the „globesity“ epidemic? Med Hypotheses 2010; 75: 250-256.

2. Flegal KM, Carroll MD, Ogden CL et al. Prevalence and trends in obesity among US adults, 1999-2008. J Amer Med Ass 2010; 303 (3): 235-241.

3. National Center for Health Statistics. Chartbook on Trends in the Health of Americans. Health, United States, 2008. Hyattsville, MD: Public Health Service. 2008.

4. Ginter E, Simko V. Adult obesity at the beginning of the 21st century: epidemiology, pathophysiology and health risk. Bratisl Lek Listy 2008; 109 (5): 224-230.

5. Allison DB, Downey M, Atkinson RL et al. Obesity as a disease: a white paper on evidence and arguments commissioned by the Council of the Obesity Society. Obesity 2008; 16 (6): 1161-1177. 
6. Brown LM, Gent L, Davis K et al. Metabolic impact of sex hormones on obesity. Brain Res 2010; 1350: 77-85.

7. Enerbäck S. Human brown adipose tissue. Cell Metab 2010; 11 (4): $248-252$.

8. Lidell ME, Enerback S. Brown adipose tissue-a new role in humans? Nat Rev Endocrinol 2010; 6: 319-325.

9. Mattson MP. Does brown fat protect against diseases of aging? Ageing Res Reviews 2010; 9: 69-76.

10. Lee P, Ho K, Fulham MJ. The importance of brown adipose tissue. New Engl J Med 2009; 361: 418.

11. Timmons JA, Pedersen BK. The importance of brown adipose tissue. New Engl J Med 2009; 361: 415-416.

12. Nedergaard J, Bengtsson T, Cannon B. Unexpected evidence for active brown adipose tissue in adult humans. Am J Physiol Endocrinol Metab 2007; 293 (2): E444-452.

13. Saito M, Okamatsu-Ogura Y, Matsushita M et al. High incidence of metabolically active brown adipose tissue in healthy adult humans. Diabetes 2009; 58 (7): 1526-1531.

14. Kozak LP. Brown fat and the myth of diet-induced thermogenesis. Cell Metab 2010; 11: 263-267.

15. Au-Yong IT,Thorn N, Ganatra $\mathbf{R}$ et al. Brown adipose tissue and seasonal variation in humans. Diabetes 2009; 58: 2583-2587.

16. Pfannenberg $C$, Werner MK, Ripkens $S$ et al. Impact of age on the relationships of brown adipose tissue with sex and adiposity in humans. Diabetes 2010; 59: 1789-1793.

17. Huang YC, Hsu CC, Huang P et al. The changes in brain metabolism in peple with activated brown adipose tissue: A PET study. Neuroimage 2010;Aug 2 (Epub ahead of print).

18. Verty AN, Allen AM, Oldfield BJ. The endogenous actions of hypothalamic peptides on brown adipose tissue thermogenesis in the rat. Endocrinology 2010; 151: 423-446.

19. Ravussin E, Kozak LP. Have we entered the brown adipose tissue renaissance? Obes Rev 2009; 10: 265-268.

20. Cypess AM, Lehman S, Williams G et al. Identification and importance of brown adipose tissue in adult humans. N Engl J Med 2009; 360: $1509-1517$.

21. Yamamoto Y, Gesta S, Lee KY et al. Adipose depots possess unique developmental gene signatures. Obesity 2010; 18: 872-878.

22. Wolf G. Brown adipose tissue: the molecular mechanism of its formation. Nutr Rev 2009; 67: 167-171.

23. Christian M, Parker MG. The engineering of brown fat. J Molec Cell Biol 2010; 2 (1): 23-25.

24. Ravussin E. The presence and role of brown fat in adult humans. Curr Diab Rep 2010; 10 (2): 90-92.

25. Seale $\mathbf{P}$, Lazar MA. Brown fat in humans: turning up the heat on obesity. Diabetes 2009; 58 (7): 1482-1484.
26. Cannon B, Nedergaard J. Brown adipose tissue: function and physiological significance. Physiol Rev 2004; 84 (1): 277-359.

27. Lowell BB, Susulic VS, Hamann A et al. Development of obesity in transgenic mice after genetic ablation of brown adipose tissue. Nature 1993; 366: 740-742.

28. Cypess AM, Lehman S, Williams G et al. Identification and importance of brown adipose tissue in adult humans. New Eng J Med 2009; 360: 1509-1517.

29. Lee P, Greenfield JR, Ho KK, Fulham MJ. A critical appraisal of prevalence and metabolic significance of brown adipose tissue in adult humans. Am J Physiol Endocrinol Metab 2010; 299 (4): E601-606.

30. van Marken Lichtenbelt WD, Vanhommerig JW, Smulders NM et al. Cold-activated brown adipose tissue in healthy men. New Eng J Med 2009; 360: 1500-1507.

31. Esterbauer H, Oberkofler H, Liu YM et al. Uncoupling protein-1 mRNA expression in obese human subjects: The role of sequence variations at the uncoupling protein-1 gene locus. J Lipid Res 1998; 39: 834-844.

32. Tanzi MC, Fare S. Adiopose tissue engineering: state of the art, recent advances and innovative approaches. Expert Rev Med Devices 2009; 6: $533-551$

33. Tseng YH, Cypess AM, Kahn CR. Cellular bioenergetics as a target for obesity therapy. Nat Rev Drug Discov 2010; 9: 465-482.

34. Miranda EJ, McIntyre IM, Parker DR et al. Two deaths attributed to the use of 2,4-dinitrophenol. J Analyt Toxicol 2006; 30 (3): 219-222.

35. Tan DX, Manchester LC, Fuentes-Broto L et al. Significance and application of melatonin in the regulation of brown adipose tissue metabolism: relation to human obesity. Obes Rev. 2010 Jun 16. [Epub ahead of print].

36. Amieux PS, McKnight GS. Cyclic nucleotides converge on brown adipose tissue differentiation. Sci Signal. 2010; 3 (104): p. pe2.

37. McKnight JR, Satterfield MC, Jobgen WS et al. Beneficial effects of L-arginine on reducing obesity: potential mechanisms and important implications for human health. Amino Acids 2010; 39 (2): 349-357.

38. Ruan X, Li Z, Zhang Y, Yang L et al. Apolipoprotein A-I possesses an anti-obesity effect associated with increase of energy expenditure and upregulation of UCP1 in brown fat. J Cell Mol Med. 2010 Feb 27. [Epub ahead of print].

39. Skarulis MC, Celi FS, Mueller E et al. Thyroid hormone induced brown adipose tissue and amelioration of diabetes in a patient with extreme insulin resistance. J Clin Endocrinol Metab 2010; 95 (1): 256-262.

40. Langin D. Recruitment of brown fat and conversion of white into brown adipocytes: strategies to fight the metabolic complications of obesity? Biochim Biophys Acta 2010; 1801 (3): 372-376.

41. Kajimura $S$, Seale $P$, Tomaru $T$ et al. Regulation of the brown and white fat gene programs through a PRDM16/CtBP transcriptional complex. Genes \& Dev 2008; 22: 1397-1409.

Received October 6, 2010. Accepted September 20, 2011. 